\title{
Trilogies of Histone Lysine Methylation as Epigenetic Landmarks of the Eukaryotic Genome
}

\author{
M. Lachner, R. Sengupta, G. Schotta, and T. Jenuwein \\ Research Institute of Molecular Pathology (IMP), The Vienna Biocenter, A-1030 Vienna, Austria
}

The past several years have been highlighted by the landmark descriptions of the genomes of several model organisms. The significance of these findings becomes even greater following the nearly full sequence assembly of the human genome. Together, these "genome projects" have shown that more complex eukaryotic model organisms have a much bigger genome than unicellular eukaryotes, although the increased "biocomplexity" is not reflected by an equivalent expansion in the number of protein-coding genes (e.g., $\sim 40,000$ in humans vs. $\sim 6,000$ in Saccharomyces cerevisiae). These results strongly suggest that biocomplexity is only in part regulated by overall gene number, but largely depends on combinatorial control triggering a vast number of gene expression patterns. In addition, mechanisms other than DNA sequence information have been used during evolution to better index and regulate the complex developmental programs and key regulatory processes, such as chromosome segregation and cell division of eukaryotic genomes.

In the nuclei of almost all eukaryotic cells, genomic DNA is highly folded and compacted with histone and nonhistone proteins in a dynamic polymer called chromatin. The discoveries that DNA methylation, nucleosome remodeling, histone modification, and noncoding RNAs can organize chromatin into accessible ("euchromatic") and inaccessible ("heterochromatic") subdomains reveal epigenetic mechanisms that considerably extend the information potential of the genetic code. Thus, one genome can generate many "epigenomes" (Fig. 1), as the fertilized egg progresses through development and translates its information into a multitude of cell fates. These epigenetic mechanisms are crucial for the function of most, if not all, chromatin-templated processes and link alterations in chromatin structure to allele-specific expression differences, developmental programming of cell lineages, chromosome segregation, DNA repair, and genome stability. The implications of epigenetic research for human biology and disease, including cancer and aging, are far reaching.

The basic repeating unit of chromatin is the nucleosome, consisting of $147 \mathrm{bp}$ of DNA wrapped around an octamer of the core histones H2A, H2B, H3, and H4 (Luger et al. 1997). Posttranslational modifications of the protruding histone amino-termini (histone "tails") were proposed 40 years ago to affect gene expression (Allfrey et al. 1964) and have since been demonstrated as important modulators of chromatin structure, culminating in the "histone code" hypothesis (Strahl and Allis 2000; Turner 2000; Jenuwein and Allis 2001).

Histone modifications include acetylation, phosphorylation, methylation (arginine and lysine), ubiquitination, and ADP ribosylation (van Holde 1988). In the last 4 years, histone lysine methylation has emerged as a central epigenetic modification (Jenuwein 2001; Zhang and
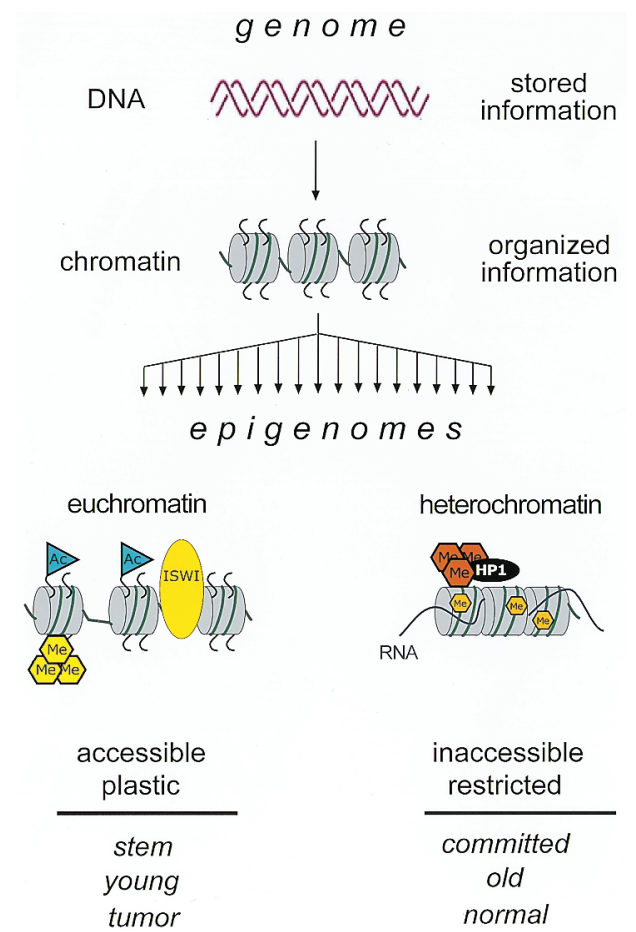

Figure 1. Genome versus epigenomes. The figure illustrates the distinction between genome (DNA sequence) and epigenome (the collective modification pattern of chromatin). Various mechanisms, including noncoding RNAs, histone modification, DNA methylation, and chromatin-remodeling activities, contribute to the generation of $>1000$ "epigenomes" from one genome template, as the fertilized egg progresses during development. Differences in the ratio between accessible ("euchromatic") and inaccessible ("heterochromatic") chromatin domains allow or restrict transcription from the underlying DNA sequence and may represent an organizing principle to regulate the use of the genetic information. The various epigenetic modifications are indicated as histone acetylation (blue flag), histone lysine methylation (green hexagons, active marks; red hexagons, repressive marks), DNA methylation (small orange hexagons), and remodeling factors (e.g., ISWI). See text for further explanation. 
Reinberg 2001; Kouzarides 2002; Fischle et al. 2003a; Lachner et al. 2003). To date there are five well described and prominently methylated lysine positions in the histone $\mathrm{H} 3$ and $\mathrm{H} 4$ tails. According to their associated functions, histone $\mathrm{H} 3$ lysine 4 (H3-K4) and lysine 36 (H3K36) methylation are classified as transcriptional "ON" marks, while histone H3 lysine 9 (H3-K9), lysine 27 (H3$\mathrm{K} 27$ ), and histone $\mathrm{H} 4$ lysine 20 (H4-K20) methylation represent "OFF" marks, which are mainly involved in the organization of repressive chromatin structures. Each of these individual lysine positions can exist in three distinct methylation states: mono-, di, and trimethylation (the "trilogies").

Here, we describe a comprehensive analysis of the presence and abundance of all possible methylation states for H3-K9, H3-K27, and H4-K20 in mammalian chromatin and in several eukaryotic model organisms. We show that selective combinations between distinct methylation states can discriminate constitutive from facultative heterochromatin. Yet other combinations of repressive methyl-lysine marks appear to be involved in Polycomb-mediated gene silencing. We also discuss implications of why there are differences in mono-, di-, and trimethylation and how distinct histone lysine methylation systems are functionally connected to impart a combinatorial histone lysine methylation pattern.

In mammalian chromatin, monomethylation and, to a larger extent, trimethylation are focally enriched at chromosomal subdomains, whereas dimethylation is not very informative for regional chromatin organization. H3-K9 methylation is a hallmark of constitutive heterochromatin and is evolutionarily conserved, although there are significant differences between its abundance in Schizosaccharomyces pombe and mammalian chromatin. H3-K27 methylation appears to indicate the emergence of multicellularity and of the Polycomb system; it is not detectable in S. pombe chromatin. H4-K20 methylation is highly abundant and the most conserved of the three repressive methyl-lysine marks. Based on its broad chromosomal presence, it may serve several functions, including gene silencing and chromatin condensation. Together, these data allow the assignment of distinct roles for repressive histone lysine methylation states and underscore the indexing potential of histone lysine methylation as epigenetic landmarks in eukaryotic chromatin.

\section{EPIGENETIC CONTROL OF EUKARYOTIC GENOMES}

Identical twins are used as the cover illustration for this 69th Cold Spring Harbor Symposium-Epigeneticsand as such underscore the power of genetics over the subtle differences that are imparted by epigenetic control. However, "You can inherit something beyond the DNA sequence. That's where the real excitement in genetics is now" (Watson 2003). Indeed, alterations in DNA methylation patterns (Bird 2002), misregulated chromatin remodeling (Klochendler-Yeivin et al. 2002; Narlikar et al. 2002), and changes in histone modifications (Schneider et al. 2002) have been linked with perturbed development and tumorigenesis (Jones and Baylin 2002) and with inefficient reprogramming of cloned mammalian embryos after nuclear transfer (Jaenisch and Bird 2003).

Genome size, and how the genetic information is partitioned in more complex organisms, is another aspect to be considered when gauging the importance of epigenetic control. A comparison between the genomes of $S$. cerevisiae (Yeast Genome Directory 1997), S. pombe (Wood et al. 2002), Drosophila melanogaster (Adams et al. 2000), and Mus musculus (Waterston et al. 2002) indicates that genome size significantly expands with the complexity of the respective organism $(\geq 1000$-fold between $S$. cerevisae and mouse), despite an only modest increase in overall gene number ( 6-7-fold between $S$. cerevisiae and mouse) (see Fig. 2). In contrast to the largely "open" genomes of the unicellular fungi, multicellular organisms have accumulated repetitive elements and noncoding regions, which, for example, in the mouse account for the majority of its DNA sequence (52\% noncoding and $44 \%$ repetitive DNA). Only $\sim 4 \%$ of the mouse genome encodes for protein function. This massive expansion of repetitive and noncoding sequences in multicellular organisms is most likely due to the incorpo-
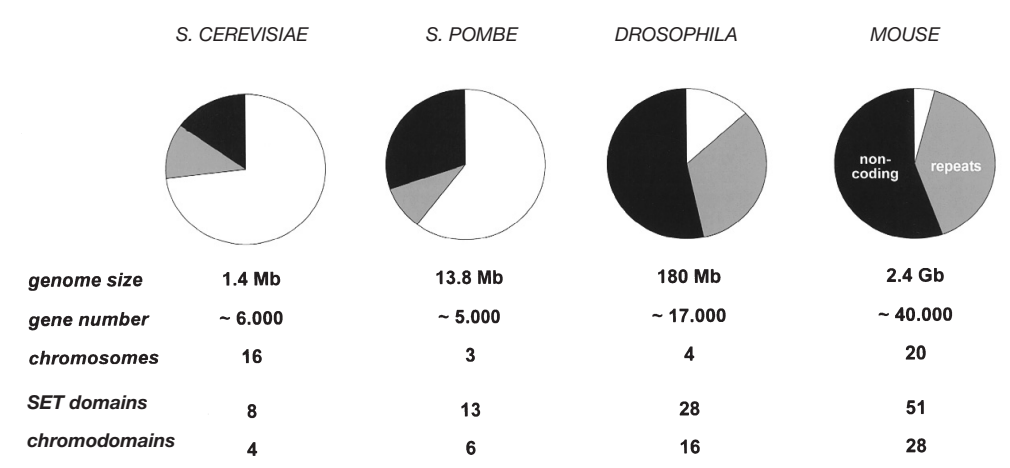

Figure 2. Genome partitioning of eukaryotic model organisms. Overview on the relative partitioning of coding (white), repetitive (gray), and noncoding (black) sequences within the genomes of S. cerevisiae, S. pombe, D. melanogaster, and M. musculus. Additionally, the respective genome sizes (in base pairs), approximate gene numbers, and chromosome numbers are indicated. All data were retrieved from published sequences (Yeast Genome Directory 1997; Adams et al. 2000; Waterston et al. 2002; Wood et al. 2002). Also listed are the approximate number of SET-domain and chromodomain proteins that are encoded by the various genomes and that have been derived by blast searches from public databases. 
ration of invasive elements, such as DNA and retrotransposons (Kazazian 2004). Although these represent a burden for coordinated gene expression programs, they also allow for genome plasticity and a certain degree of stochastic gene regulation (Han et al. 2004).

S.cerevisiae with its "open" genome lacks almost all of the known components for epigenetic gene silencing, such as the RNAi machinery, repressive histone lysine methylation systems (see below), and DNA methylation. Intriguingly, during evolution from $S$. cerevisiae to mouse, there is a similar increase ( $\sim 6-7$ - fold) in SET-domain and chromodomain genes, as there is for overall gene number. Putative HMTases (i.e., proteins that carry the signature SET domain) expand from 8 in $S$. cervisiae to 51 in mouse (see Fig. 2). For chromodomain (i.e., a module that has been shown to confer methyl-binding activity) proteins, there is a similar increase from 4 in $S$. cerevisiae to $\sim 28$ in mice.

Histone lysine methylation systems generally consist of a SET-domain enzyme and a chromodomain adaptor. Since there are only five prominent methyl-lysine residues in the histone $\mathrm{H} 3$ and $\mathrm{H} 4$ tails, each methylatable lysine can potentially be targeted by multiple HMTases. For example, the H3-K9 position is a substrate for the activity of at least three distinct HMTase systems in mammalian chromatin (Suv39h1 and Suv39h2: O'Carroll et al. 2000; Rea et al. 2000; G9a and Glp1: Ogawa et al. 2002; Tachibana et al. 2002; ESET: Yang et al. 2002). Thus, for the "closed" genomes of complex multicellular organisms, various repressive HMTase systems may interact with the other silencing mechanisms, such as the RNAi machinery and DNA methylation, to restrict aberrant transcriptional activity in a very effective manner. This silencing synergy is also important to prevent remobilization of transposons and contributes to the specialized chromatin organization at pericentric and telomeric regions in these highly partitioned genomes.

\section{FUNCTIONAL ROLES OF HISTONE LYSINE MONO-, DI-, AND TRIMETHYLATION}

Early biochemical analyses have indicated that histone lysine positions can be mono-, di-, or trimethylated (Paik and Kim 1971; DeLange and Smith 1973). These findings have recently been substantiated by functional studies in S. cerevisiae (Santos-Rosa et al. 2002) and by mass-spectrometry analyses of bulk histone preparations (Peters et al. 2003; Rice et al. 2003; L. Zhang et al. 2003). Additionally, the structural resolutions of the SET domains of various HMTases have revealed the molecular mechanism of substrate and product specificity for these enzymes (B. Xiao et al. 2003; X. Zhang et al. 2003). Based on these three-dimensional structures, putative HMTases can be predicted to be mono-, di-, or trimethylating enzymes by comparing the primary amino acid sequence of their associated SET domain. Further, directed mutational analyses allow the conversion of a monomethylating enzyme to become a trimethylating HMTase by engineering a slightly larger catalytic pocket via the exchange of a Tyr with a Phe (X. Zhang et al. 2003). Despite these significant advances, the question as to the physiological relevance of why there are three distinct histone lysine methylation states remains largely unsolved.

One possibility is that the distinct methylation states would generate different affinities for chromatin-associated proteins. The structural resolution of several chromodomain proteins, such as HP1 (Jacobs and Khorasanizadeh 2002; Nielsen et al. 2002) and Polycomb (Fischle et al. 2003c; Min et al. 2003) have allowed predictions that discriminate position-specific binding toward H3-K9 or H3-K27 methylation. HP1 displays a similar in vitro affinity for $\mathrm{H} 3-\mathrm{K} 9$ dimethylation and trimethylation, while its $K_{\mathrm{d}}$ is significantly reduced for H3-K9 monomethylation (Fischle et al. 2003c). An H3K9 monomethyl mark is not sufficient for in vivo HP1 binding to pericentric heterochromatin (Peters et al. 2003). Chromatin recruitment of HP1 may be facilitated by nucleosomal surfaces (Nielsen et al. 2001; Meehan et al. 2003) and an RNA affinity (Maison et al. 2002; Muchardt et al. 2002). Similarly, the $K_{\mathrm{d}}$ of Polycomb for H3-K27 trimethylation is significantly stronger than for H3-K27 dimethyl or monomethyl peptides (Fischle et al. $2003 c)$. These in vitro data reflect only potential in vivo affinities and, in particular for Polycomb binding, additional marks, such as H3-K9 (Czermin et al. 2002; L. Ringrose and R. Paro, pers. comm.) and possibly histone H1b-K26 methylation (Kuzmichev et al. 2004), may be required. Dimerization of HP1 (Brasher et al. 2000) or of Polycomb (Min et al. 2003) proteins could further stabilize histone tail interactions. Thus, there are known chromatin binders for dimethyled and trimethylated histone lysine residues, but none for a monomethylated substrate. Also, no mutational analyses have been described that would convert chromodomain affinity from tri- to di- to monomethyl binding.

A second possibility is that the distinct methylation states could reflect the stability of histone lysine methylation. There is currently no biochemical data on differential turnover rates of mono-, di-, and trimethylation of the various histone lysine positions. Immunofluorescence analyses with position- and state-specific methyl-lysine histone antibodies (see below) have suggested the trimethylated state as a robust mark, because it appears mitotically stable at pericentric heterochromatin (H3-K9 and H4-K20 trimethylation) (Peters et al. 2003; Schotta et al. 2004) or at the Xi (H3-K27 trimethylation) (Plath et al. 2003; Silva et al. 2003; Okamoto et al. 2004). Recent kinetic studies using an inducible Xist transgene have indicated prolonged association of $\mathrm{H} 3-\mathrm{K} 27$ trimethylation at the Xi after removal of Xist (Kohlmaier et al. 2004). In addition, nuclear transfer experiments revealed the persistent presence of somatic-specific histone lysine trimethylation patterns in cloned embryos (Santos et al. 2003).

There is yet another intriguing possibility for a more stable propagation of the trimethylated state. H3-K9 dimethylation and $\mathrm{H} 3-\mathrm{S} 10$ phosphorylation have been shown to be mutually exclusive (Rea et al. 2000). Since histone modifications do not appear to occur in isolation (Fischle et al. 2003b), H3-K9 trimethylation could be 


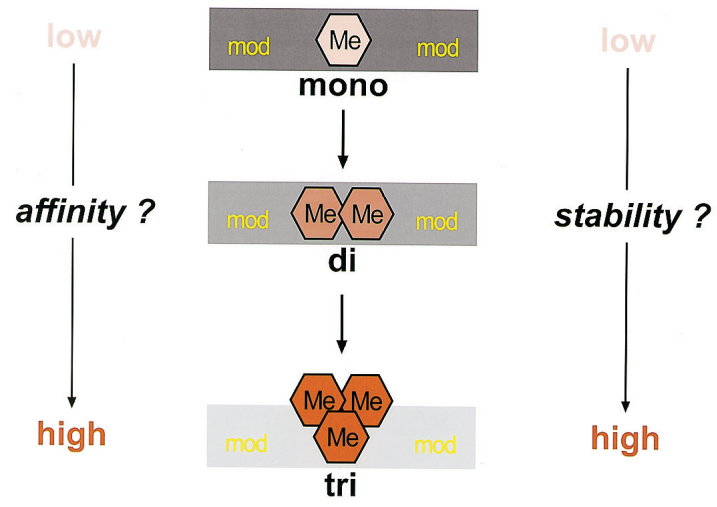

Figure 3. Potential roles of histone lysine mono-, di-, and trimethylation. Selective lysine positions in histone tails can be mono-, di-, or trimethylated, as illustrated by increasing color contrast of methyl-lysine marks (red hexagons). The distinct methylation states could modulate the affinity toward methyl-lysine binding proteins, differ in their turnover rates and stability, or display various potentials to interfere with antagonistic modifications (yellow mod) at a histone modification cassette (Fischle et al. 2003b) or in a given histone tail segment (gray bars). Decreasing shades of gray bars would reflect that a trimethylated state may impair antagonistic modifications at a higher efficiency as compared to monomethylation or dimethylation.

more efficient in preventing antagonistic marks such as acetylation or phosphorylation within the same modification cassette on a given histone segment (Fig. 3). A methyl/phospho switch for HP1 binding has been proposed, predicting impaired HP1 association if the H3-K9 methylated histone tail is additionally phosphorylated at H3-S10 (Fischle et al. 2003b). It is conceivable that H3K9 trimethylation could inhibit substrate recognition of H3-S10-specific kinases more efficiently than H3-K9 monomethylation or dimethylation (see Fig. 3) (e.g., by presenting a stronger sterical hindrance). According to this model, H3-K9 trimethylated chromosomal regions would maintain the recruitment signal for HP1 and hence propagate a distinct chromatin structure in a more stable manner. Recently, phosphoacetylation of histone H3 peptides has been shown to weaken the affinity of HP1 toward an H3-K9 dimethylated position (Mateescu et al. 2004).

\section{HISTONE LYSINE METHYLATION PATTERNS ARE EPIGENETIC LANDMARKS OF EUKARYOTIC CHROMATIN}

To examine the in vivo distribution of distinct histone lysine methylation states in native chromatin, we developed a series of position-specific methyl-lysine antibodies against $\mathrm{H} 3-\mathrm{K} 9, \mathrm{H} 3-\mathrm{K} 27$, and $\mathrm{H} 4-\mathrm{K} 20$ that additionally discriminate between mono-, di-, and trimethylation (Peters et al. 2003; Perez-Burgos et al. 2004; Schotta et al. 2004). Immunofluorescence analysis of wild-type and Suv39h double null (Suv39h dn) female mouse embryonic fibroblasts (iMEFs) (Fig. 4) indicated a rather speckled and noninformative distribution for the dimethylated

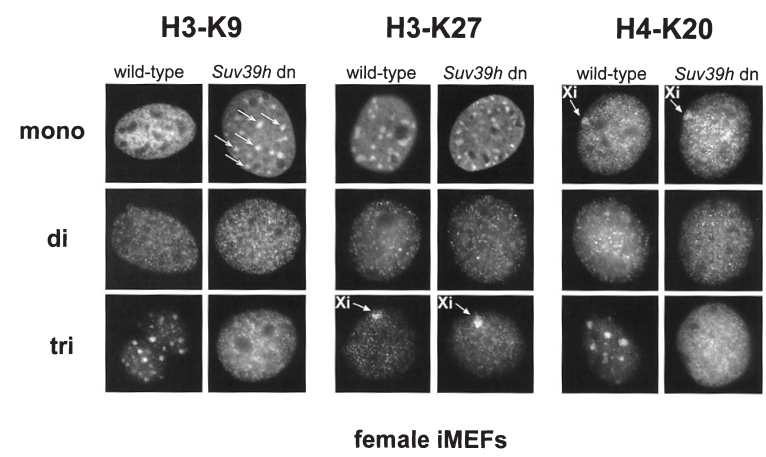

Figure 4. Repressive histone lysine methylation states are epigenetic landmarks in mouse interphase chromatin. Female wildtype and Suv39h dn iMEFs were stained with methyl-lysine histone antibodies that discriminate mono-, di-, and trimethylation of H3-K9 (Peters et al. 2003; Perez-Burgos et al. 2004), H3-K27 (Peters et al. 2003; Perez-Burgos et al. 2004), and H4-K20 (Schotta et al. 2004). Foci of pericentric heterochromatin that were visualized by costaining with DAPI (not shown) are decorated by H3-K9 tri-, H3-K27 mono-, and H4-K20 trimethylation in wild-type nuclei. In addition, the inactive X chromosome (Xi) is enriched for $\mathrm{H} 3-\mathrm{K} 27$ trimethylation and $\mathrm{H} 4-\mathrm{K} 20$ monomethylation. The occurrence of $\mathrm{H} 3-\mathrm{K} 9$ monomethylation at pericentric heterochromatin in $S u v 39 h$ dn nuclei is indicated by multiple arrows.

state throughout the interphase nucleus. By contrast, the monomethyl and trimethyl specific antibodies revealed selective enrichment with distinct subnuclear chromatin domains.

Using DAPI staining (data not shown) to visualize the foci of pericentric heterochromatin, wild-type iMEFs display enrichment for H3-K9 tri-, H3-K27 mono-, and H4K20 trimethylation at constitutive heterochromatin (Fig. 4). In addition, H3-K9 monomethylation is excluded from these pericentric regions. In Suv39h dn cells, H3-K9 trimethylation is lost from pericentric heterochromatin, which instead accumulates prominent H3-K9 monomethyl signals (see multiple arrows in Fig. 4). These data suggest that H3-K9 monomethylation (mediated by a currently unidentified H3-K9 monomethylase) could serve as an intermediate in vivo substrate for the trimethylating Suv39h enzymes. Strikingly, the loss of Suv39h activity also abolishes the staining for H4-K20 trimethylation at pericentric heterochromatin, although the H4-K20 position is not an intrinsic substrate for these enzymes. Recent data have identified a silencing pathway in which nucleosome-specific H4-K20 trimethylating enzymes (Suv4-20h HMTases) act downstream of Suv39hdependent H3-K9 trimethylation (Schotta et al. 2004).

In these female iMEFs, the Barr body that represents the inactive $\mathrm{X}$ chromosome (Xi) can also be visualized as a single focus that is largely localized at the nuclear periphery (see arrows indicating Xi in Fig. 4). Intriguingly, the $\mathrm{Xi}$ is decorated by a different combination of methyllysine marks, which include H3-K27 trimethylation and H4-K20 monomethylation. This staining pattern remains unaltered in wild-type and Suv39h dn cells, consistent with distinct HMTase systems to control epigenetic im- 
prints of the Xi (Plath et al. 2003; Silva et al. 2003; Kohlmaier et al. 2004; Okamoto et al. 2004; see below).

Thus, our comprehensive analysis on all nine repressive histone lysine methylation states (the three "trilogies") has revealed selective patterns to index distinct chromosomal subdomains. Constitutive heterochromatin at pericentric regions is characterized by a combination of H3-K9 tri-, H3-K27 mono-, and H4-K20 trimethylation, whereas the signature for facultative heterochromatin at the $\mathrm{Xi}$ consists of the two prominent marks H3-K27 trimethylation and H4-K20 monomethylation.

\section{HMTase NETWORKS INDEX HETEROCHROMATIN, THE Xi AND Polycomb SILENCING}

Based on the above data, induction of the observed histone lysine methylation pattern at constitutive heterochromatin would involve interplay of (at least) four different HMTases. Whereas the function of the predicted H3-K27 monomethylase is unresolved, another monomethylase appears required to prepare the histone $\mathrm{H} 3$ tail via H3-K9 monomethylation for subsequent substrate recognition by the trimethylating Suv39h enzymes (Peters et al. 2003). Suv39h-dependent H3-K9 trimethylation then provides a binding platform for the HP1 proteins and recruitment of the nucleosome-specific, trimethylating Suv4-20h HMTases (Schotta et al. 2004). None of the proposed H3-K27 and H3-K9 monomethylating enzymes are currently identified, nor do we know the nature of any of the putative H3-K27 monomethyl and H4-K20 trimethyl-lysine binders (Fig. 5).

At the $\mathrm{Xi}$, the presence of $\mathrm{H} 4-\mathrm{K} 20$ monomethylation requires the activity of an unidentified H4-K20 monomethylase (Kohlmaier et al. 2004). In addition, H3$\mathrm{K} 9$ dimethylation may accumulate at the $\mathrm{Xi}$ during the cell cycle (K. Plath and B. Panning, pers. comm.), where it could provide a transient signal for HP1 (Chadwick and Willard 2003). The most robust modification at facultative heterochromatin, however, is H3-K27 trimethylation, which is mediated by the Polycomb group and SETdomain enzyme Ezh (Plath et al. 2003; Silva et al. 2003; Okamoto et al. 2004). There is currently no reported association of other Polycomb group proteins with the $\mathrm{Xi}$, although not all components of the Polycomb system have been examined. It is also unclear how the predicted H4-K20 monomethylase would interact with an Xi-specific Ezh2 complex to induce a combinatorial histone lysine methylation pattern.

For Polycomb-mediated gene silencing, the initial data suggested Ezh2-dependent H3-K9 and H3-K27 methylation as important signals, with a preference for H3-K27 trimethylation (Cao et al. 2002; Czermin et al. 2002; Kuzmichev et al. 2002; Müller et al. 2002). Ezh2 is an HMTase that displays activity only upon complex formation with the other Polycomb-group proteins $\mathrm{Su}(\mathrm{z}) 12$ and Eed. Biochemical fractionation has recently identified several distinct Ezh2 complexes that contain different amino-terminal isoforms of Eed: PRC2 (Eed1) and PRC3 (Eed 3/4) (Kuzmichev et al. 2004). Strikingly, these complexes target mutually exclusive histone substrates when tested in a nucleosomal context containing linker histones. In this setting, PRC2 is inactive toward histone H3, and instead trimethylates the linker histone $\mathrm{H} 1 \mathrm{~b}$ at the $\mathrm{K} 26$ position. In contrast, PRC3 has a preference for $\mathrm{H} 3-$ $\mathrm{K} 27$ trimethylation, but only if the nucleosomal templates lack the linker histones. From these data, Ezh2 appears as a very promiscuous HMTase whose substrate specificity would be context dependent. It would thus not be inconceivable that distinct Ezh complexes at different developmental options could target H3-K9, H3-K27, and/or H1bK26 positions, which together may generate a robust combinatorial signal to recruit Polycomb complexes for stable gene silencing.

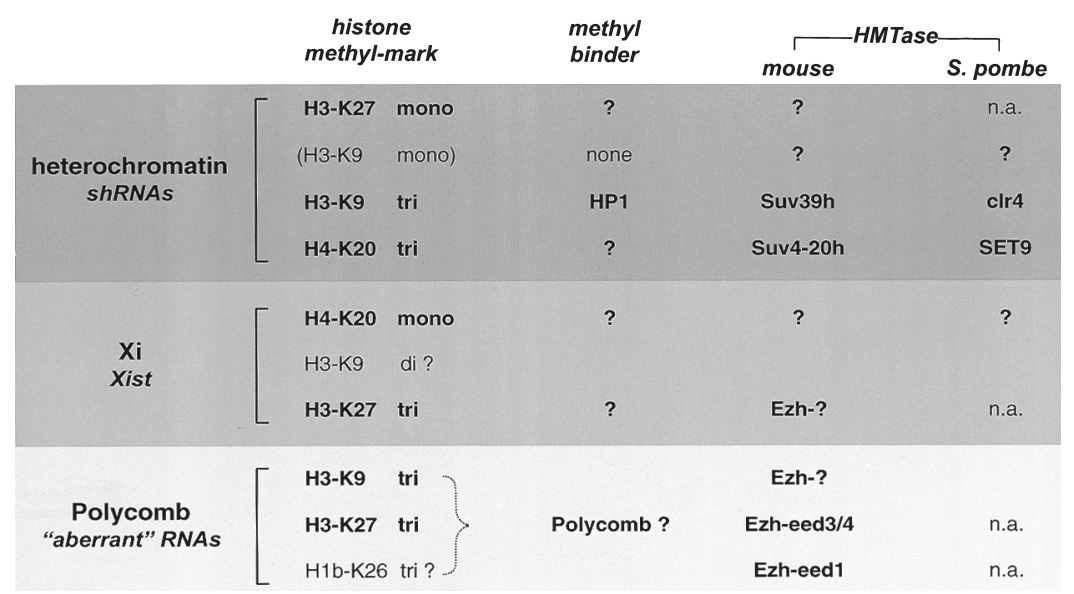

Figure 5. HMTase networks for heterochromatin, the Xi, and Polycomb. This figure summarizes combinations of mammalian histone lysine methylation marks that are enriched at distinct chromatin domains, such as pericentric heterochromatin or the inactive $\mathrm{X}$ chromosome (see Fig. 4), or that could provide a combinatorial signal to stabilize Polycomb complexes at their target regions. Confirmed histone lysine-methyl marks are shown in bold. Also indicated are known methyl-lysine binding partners and the relevant HMTases (with their $S$. pombe homologs, where appropriate). The Polycomb group HMTase Ezh only works in a complex and appears as a promiscuous enzyme whose substrate specificity is directed via interaction with different eed isoforms (Kuzmichev et al. 2004). Whether additional eed isoforms, eed modifications, or other Ezh complex components may alter substrate specificity toward $\mathrm{H} 3-\mathrm{K} 9$ or facilitate recruitment at the Xi is currently unresolved (Ezh-?). n.a.: not applicable. 


\section{HISTONE LYSINE METHYLATION STATES IN MOUSE VERSUS S. POMBE}

At mouse and Drosophila (Schotta et al. 2004) heterochromatin, we have uncovered a sequential silencing pathway for H3-K9 and H4-K20 trimethylation. To address whether this link may also be operational in unicellular eukaryotes, we analyzed histone lysine methylation states in $S$. pombe. In this organism, the Suv39h/HP1 methylation system is represented through the Clr4/Swi6 homologs, and the Suv4-20h HMTases are conserved via the SET9 enzyme (see Fig. 5, and above). Further, the enrichment of Clr4-mediated H3-K9 methylation at the outer centromeric repeats and at the mating type loci has been demonstrated by ChIP data (Nakayama et al. 2001; Noma et al. 2001; Volpe et al. 2003).

To examine the relative abundance of repressive histone lysine methylation marks, we probed bulk histone preparations from wild-type and clr $4 \Delta S$. pombe strains with our panel of position- and state-specific methyl-lysine histone antibodies (Fig. 6). Nuclear extracts from wild-type and Suv39h dn iMEFs were used as a reference. To demonstrate the quality of the histone preparations from $S$. pombe, we first hybridized the protein blots with H3-K4-specific antibodies. Similar and abundant levels for H3-K4 mono-, di-, and trimethylation could be visualized, consistent with this active mark being a prominent modification in fission yeast (Noma and Grewal 2002). Surprisingly, there were no detectable signals with any of the H3-K9 state-specific antibodies, despite the documented presence of $\mathrm{H} 3-\mathrm{K} 9$ dimethylation by ChIP analyses (Nakayama et al. 2001; Noma et al. 2001; Volpe et al. 2003). These data indicate a very low abundance for H3K9 methylation and suggest that this mark may be locally enriched only in $S$. pombe chromatin. For the three H3K27 methylation states, we also failed to detect any signals. This would be consistent with the absence of the Polycomb system in unicellular eukaryotes, and there is no fission yeast SET-domain gene with apparent sequence homology to the Ezh HMTases.

In striking contrast, however, H4-K20 mono-, di-, and trimethylation states are very prominent in the $S$. pombe histone preparations. Interestingly, there is a moderate reduction in H4-K20 tri-, but not mono- or dimethylation levels, in clr $4 \Delta$ mutants, similar to that observed in wildtype versus $S u v 39 h$ dn nuclear extracts (Fig. 6, right panel). These data suggest that a fraction of H4-K20 trimethylation could be dependent on $\mathrm{Clr} 4$ function, indicating a possible evolutionary conservation between H3K9 and H4-K20 methylation systems from S. pombe to mammals.

\section{EVOLUTIONARY CONSERVATION OF HISTONE LYSINE METHYLATION STATES}

The surprising differences in the selective abundance of repressive histone lysine methylation states in $S$. pombe versus mouse prompted us to compile a summary of histone lysine methylation "trilogies" in several other model organisms, including S. cerevisiae, Arabidopsis thaliana, and Drosophila (Fig. 7). For simplification, we focused only on prominent methyl-lysine positions in the histone tails and divided this summary into "ON" marks (H3-K4 and H3-K36) and "OFF" marks (H3-K9, H3$\mathrm{K} 27$, and $\mathrm{H} 4-\mathrm{K} 20$ ).

$S$. cerevisiae chromatin does not carry any of the currently described "OFF" marks. This is consistent with the lack of the other components that have been implicated in the epigenetic hierarchy of gene silencing, such as the RNAi machinery and DNA methylation. For its few repressed chromatin domains (telomeres and the mating-type loci), $S$. cerevisiae has developed a unique silencing system that is mainly based on the SIR proteins (Grunstein 1997; Gasser and Cockell 2001). Consistent with the "activated" genome of S. cerevisiae (see Fig. 2), the "ON" marks are present (Briggs et al. 2001), including H3-K4 trimethylation (Santos-Rosa et al. 2002), H3K36 methylation (Strahl et al. 2002; T. Xiao et al. 2003), and the antisilencing function imparted by H3-K79 methylation (Feng et al. 2002; van Leeuwen et al. 2002).

Although the $S$. pombe epigenome also has only a limited amount of repressed chromosomal regions (centromeres, telomeres, and mating-type loci), it contains a
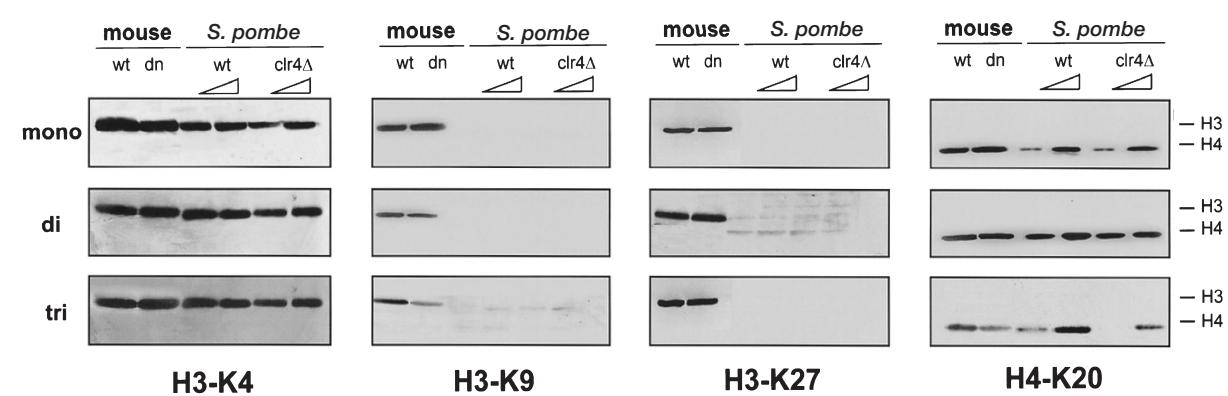

Figure 6. Protein blot analysis for histone lysine methylation states in mouse versus $S$. pombe extracts. Protein blots of nuclear extracts $(12 \mu \mathrm{g})$ from wild-type (wt) and $S u v 39 h \mathrm{dn}$ iMEFs and of acid-extracted histone preparations from wt and clr $4 \Delta S$. pombe strains ( $25 \mu \mathrm{g}$ and $50 \mu \mathrm{g}$, respectively) were probed with methyl-lysine histone antibodies directed against mono-, di-, and trimethylation of H3-K4 (Upstate Biotech), H3-K9 (Peters et al. 2003; Perez-Burgos et al. 2004), H3-K27 (Peters et al. 2003; Perez-Burgos et al. 2004), and H4-K20 (Schotta et al. 2004). The relative positions of histone $\mathrm{H} 3$ and $\mathrm{H} 4$ are indicated on the far right. 


\begin{tabular}{|c|c|c|c|c|c|c|c|}
\hline & & & S. cerevisiae & S. pombe & Arabidopsis & Drosophila & mouse \\
\hline \multirow{2}{*}{ ON } & H3-K4 & $\begin{array}{c}\text { mono } \\
\text { di } \\
\text { tri }\end{array}$ & $\begin{array}{l}+ \\
+ \\
+\end{array}$ & $\begin{array}{l}+ \\
+ \\
+\end{array}$ & $\begin{array}{l}+ \\
+ \\
+\end{array}$ & $\begin{array}{l}+ \\
+ \\
+\end{array}$ & $\begin{array}{l}+ \\
+ \\
+\end{array}$ \\
\hline & H3-K36 & $\begin{array}{c}\text { mono } \\
\text { di } \\
\text { tri }\end{array}$ & $\begin{array}{l}+ \\
+ \\
\text { nd }\end{array}$ & $\begin{array}{l}\text { nd } \\
+ \\
+\end{array}$ & $\begin{array}{l}\text { nd } \\
+ \\
+\end{array}$ & $\begin{array}{l}+ \\
+ \\
+\end{array}$ & $\begin{array}{l}+ \\
+ \\
+\end{array}$ \\
\hline \multirow{3}{*}{ OFF } & H3-K9 & $\begin{array}{c}\text { mono } \\
\mathrm{di} \\
\text { tri }\end{array}$ & : & $\begin{array}{c}? \\
(+) \\
(+)\end{array}$ & $\begin{array}{l}+ \\
+ \\
?\end{array}$ & $\begin{array}{c}+ \\
+ \\
(+)\end{array}$ & $\begin{array}{l}+ \\
+ \\
+\end{array}$ \\
\hline & H3-K27 & $\begin{array}{c}\text { mono } \\
\text { di } \\
\text { tri }\end{array}$ & : & : & $\begin{array}{l}+ \\
+ \\
\text { nd }\end{array}$ & $\begin{array}{l}+ \\
+ \\
+\end{array}$ & $\begin{array}{l}+ \\
+ \\
+\end{array}$ \\
\hline & H4-K2O & $\begin{array}{c}\text { mono } \\
\text { di } \\
\text { tri }\end{array}$ & : & $\begin{array}{l}+ \\
+ \\
+\end{array}$ & $\begin{array}{l}+ \\
+ \\
+\end{array}$ & $\begin{array}{l}+ \\
+ \\
+\end{array}$ & $\begin{array}{l}+ \\
+ \\
+\end{array}$ \\
\hline
\end{tabular}

Figure 7. Evolutionary conservation of histone lysine methylation trilogies. Summary of available data on the presence and abundance of mono-, di-, and trimethylation (the "trilogies") at the H3-K4 and H3-K36 ("ON" marks) or at the H3-K9, H3-K27, and H4K20 ("OFF" marks) positions in S. cerevisiae, S. pombe, A. thaliana, D. melanogaster, and M. musculus chromatin. +: abundant presence; -: undetectable levels; $(+)$ : this given methyl-lysine mark has been detected only by ChIP analyses or is underrepresented with respect to the other methylation states at this specific lysine position; ?: the data on this methyl-lysine mark are not confirmed. Information on some distinct methylation states was not available. nd: not done.

This summary integrates data from immunofluorescence, protein blot, ChIP, and mass spectrometry analyses, based on the following references: S. cerevisiae; H3-K4 (Briggs et al. 2001; Santos-Rosa et al. 2002); H3-K36 (Strahl et al. 2002; B. Strahl, pers. comm.); S. pombe, H3-K4 (see Fig. 6); H3-K36 (R. Sengupta and T. Jenuwein, unpubl.); H3-K9 (Nakayama et al. 2001; Noma et al. 2001; Volpe et al. 2003; M. Portoso and R. Allshire, pers. comm.; S. Grewal, pers. comm.); H3-K27 and H4-K20 (see Fig. 6); A. thaliana, H3-K4; and H3-K36 (A. Fischer and G. Reuter, pers. comm.); H3-K9 (Jasencakova et al. 2003; Jackson et al. 2004); H3K27 (Bastow et al. 2004; Sung and Amasino 2004; A. Fischer and G. Reuter, pers. comm.); H4-K20 (A. Fischer and G. Reuter, pers. comm.); D. melanogaster; H3-K4 (G. Reuter, pers. comm.); H3-K36 (S. Kubicek and T. Jenuwein, unpubl.); H3-K9 and H3-K27 (A. Ebert et al., in prep.); H4-K20 (Schotta et al. 2004); M. musculus and H3-K4 (see Fig. 6); H3-K36 (S. Kubicek and T. Jenuwein, unpubl.); H3-K9 and H3-K27 (Peters et al. 2003); and H4-K20 (Schotta et al. 2004). Additional data used to compile this summary are available from R. Sengupta and T. Jenuwein (unpubl.).

subset of the "OFF" marks. In addition to a local enrichment of H3-K9 dimethylation at these silenced domains (Nakayama et al. 2001; Noma et al. 2001; Volpe et al. 2003), some signals for H3-K9 trimethylation may also be present at the outer centromeric regions (S. Grewal, pers. comm.). In contrast, there is no proven evidence for H3-K9 monomethylation (M. Portoso and R. Allshire, pers. comm.). Also, none of the three H3-K27 methylation states can be detected, consistent with the absence of Polycomb function in fission yeast. In Neurospora crassa, H3-K9 trimethylation has been shown to be important to direct DNA methylation (Tamaru et al. 2003). Intriguingly, H4-K20 methylation represents the most prominent "OFF" mark in $S$. pombe, including all three methylation states at similar abundance. However, H4K20 methylation may not be restricted to a role in gene repression, since loss of H4-K20 methylation in set $9 \Delta$ mutants does not appear to cause silencing defects, but rather results in increased sensitivity toward DNA damage (S. Saunders, R. Allshire, and T. Kouzarides, pers. comm.).

In A. thaliana chromatin, all "OFF" marks, including H3-K27 are subject to methylation, which is consistent with described Polycomb function and H3-K27-mediated gene repression at developmentally regulated loci (Bastow et al. 2004; Sung and Amasino 2004). However, there may be significant differences as compared to mammalian chromatin with respect to the full definition of the trimethylated state, as A. thaliana apparently lacks H3$\mathrm{K} 9$ trimethylation at heterochromatic subdomains (Jasencakova et al. 2003; Jackson et al. 2004), and there are no reported data for the presence of H3-K27 trimethylation. This does not exclude local accumulation of H3-K9 and/or H3-K27 trimethylation at distinct targets or selected chromatin regions (A. Fischer and G. Reuter, pers. comm.), particularly if synergisms between some of the corresponding HMTase systems may be impaired.

Drosophila chromatin displays the full spectrum of all repressive histone lysine methylation states. Although $\mathrm{H} 3-\mathrm{K} 9$ dimethylation is most prominent at pericentric heterochromatin and dominates the trimethylated state, $\mathrm{H} 3-\mathrm{K} 9$ trimethylation is focally enriched at the core of the chromocenter (A. Ebert et al., in prep.). All three H3-K27 methylation states are present at the chromosomal arms and their accumulation is collectively governed by the Polycomb group HMTase E(z). H4-K20 trimethylation is broadly dispersed over many chromosomal regions but selectively enriched at the chromocenter, where it is required for modulation of position effect variegation (PEV) (Schotta et al. 2004).

Finally, mammalian chromatin exhibits abundant mono-, di-, and trimethylation of all examined repressive histone lysine positions (see Fig. 4). This probably reflects the need to strenghten functional interactions between the major silencing systems of the RNAi machinery, histone lysine methylation, and DNA methylation in the efficient epigenetic control of large parts of their "closed" genomes (see Fig. 2). Mass-spectrometry analyses have indicated that the sum of mono-, di-, and trimethylation accounts for $>50 \%$ of bulk histones in mouse embryonic stem cell extracts to be methylated at the $\mathrm{H} 3-\mathrm{K} 9$ or $\mathrm{H} 3-\mathrm{K} 27$ positions (Peters et al. 2003). 


\section{SUMMARY AND OUTLOOK}

We have examined the three repressive "trilogies" for H3-K9, H3-K27, and H4-K20 mono-, di-, and trimethylation. We have shown that all of these histone lysine methylation states are present in mammalian chromatin and that they can index selective chromatin regions in a combinatorial manner. In addition, we provided evidence for a functional interplay of the associated HMTase systems. Together with the data on the evolutionary conservation, it is possible to assign the following major roles for the distinct histone lysine methylation marks.

H3-K9 methylation is largely, although not abundantly, conserved from $S$. pombe to mammals and serves as a hallmark for gene silencing and the formation of heterochromatic regions. $\mathrm{H} 3-\mathrm{K} 9$ methylation can induce a repressed chromatin domain primarily at repetitive sequence elements, where its HMTase systems are likely to be recruited via short heterochromatic RNAs (shRNAs, see Fig. 5). At pericentric heterochromatin, the interplay of shRNAs and H3-K9 methylation (and of DNA methylation in the appropriate organisms) is important to safeguard centromere function and chromosome segregation.

H3-K27 methylation could indicate the emergence of multicellularity and cell-type differentiation, as it is absent in both budding and fission yeast. The presence of H3-K27 methylation signals appears tightly coupled to the existence of the Polycomb system, which is involved in lineage commitment and in coregulating the stability of gene expression programs. H3-K27 trimethylation is also a prominent epigenetic imprint for facultative heterochromatin at the $\mathrm{Xi}$, where it is targeted via the noncoding Xist RNA. Based on the recurrent parallels of RNAdependent recruitment of HMTase systems, it is conceivable that Polycomb-mediated "transcriptional memory" could also be triggered by an RNA moiety that may comprise "aberrant" or stalled transcripts (see Fig. 5) from developmentally regulated promoters.

H4-K20 methylation is highly conserved and most abundant across epigenetic model organisms ranging from $S$. pombe to mammals. Although H4-K20 trimethylation is enriched at pericentric heterochromatin, where it appears to be dependent on preexisting H3-K9 trimethylation, it is also present at many other chromosomal locations. Based on its high conservation and broad distribution, H4-K20 methylation is probably involved in several important functions, ranging from mitotic chromatin condensation (Fang et al. 2002; Nishioka et al. 2002; Rice et al. 2002) to transcriptional regulation and to sensing chromatin damage and probably also DNA repair. A crucial function for H4-K20 methylation in the structural organization of chromatin is further suggested by the positioning of H4-K20 at the outer boundary of the nucleosome (Luger et al. 1997; Luger 2003), where targeted modifications or recruitment of chromatin-associated factors and linker histones could trigger transitions from the $11-\mathrm{nm}$ to a $30-\mathrm{nm}$ chromatin fiber.

Although the above examples highlight significant advances in our understanding of the functional implications of histone lysine methylation, many questions remain. These include demonstrations on whether distinct histone marks can in general be assigned with specific bi- ological roles, what their modification-dependent binding partners are, and how combinations of these marks may affect downstream events - all of these notions represent predictions from the "histone code" hypothesis. To examine combinatorial signals of histone lysine methylation and of other histone modifications, high-resolution mass spectrometry analyses on purified nucleosomes or on enriched chromosomal subdomains will be required, but will also pose a major challenge. Further, there are crucial questions in defining the mechanisms that confer inheritance and propagation of epigenetic information, important lines of research that may possibly lead to a molecular explanation for the distinction between germ cells versus somatic cells and to a better understanding of the nature of pluripotency. Will there be an enzyme(s) that actively removes histone lysine methyl marks, or what are the alternative mechanisms to erase or stabilize chromatin imprints during continuous rounds of cell division? The functional connections between the RNAi machinery, histone lysine methylation, and DNA methylation will continue to provide exciting insights into normal and perturbed development. It is also conceivable that differences in the relative abundance between distinct histone lysine methylation states, such as the apparent underrepresentation of H3-K9 and H3-K27 trimethylation in $S$. pombe and $A$. thaliana (see Fig. 7), may reflect the greater proliferative and regenerative potential in these organisms, as compared to the more restricted developmental programs of metazoan systems.

Thus, the detailed analysis on the three repressive "trilogies" of H3-K9, H3-K27, and H4-K20 methylation has not only broadened our views on the range of chromatin modifications, but also promises to yield future insights into many basic questions of epigenetic control, ranging from cell differentiation, stem cell plasticity, and regeneration to tumorigenesis and even aging.

\section{ACKNOWLEDGMENTS}

We are particularly indebted to Manuela Portoso and Robin Allshire for $S$. pombe strains and advice on protein blot analysis with fission yeast extracts; to Andreas Fischer, Anja Ebert, and Gunter Reuter for their expert help in defining histone lysine methylation states in Drosophila and A. thaliana chromatin; and to Upstate Biotechnology for development and exchange of some of the described methyl-lysine histone antibodies. We would like to thank Danny Reinberg, Robin Allshire, Tony Kouzarides, Shiv Grewal, Brian Strahl, Barbara Panning, and Gunter Reuter for allowing us to cite work prior to its publication. We are further grateful to Joost Martens, Stefan Kubicek, and Roddy O'Sullivan for contributing some of their unpublished data, and to all other members of the Jenuwein laboratory for their continuous interest and enthusiasm on histone lysine methylation systems. Research in the laboratory of T.J. is supported by the IMP through Boehringer Ingelheim and by grants from the Vienna Economy Promotion Fund, the European Union (EU-network HPRN-CT 2000-00078), and the Austrian GEN-AU initiative, which is financed by the Austrian Ministry of Education, Science, and Culture. 


\section{REFERENCES}

Adams M.D., Celniker S.E., Holt R.A., Evans C.A., Gocayne J.D., Amanatides P.G., Scherer S.E., Li P.W., Hoskins R.A., Galle R.F., George R.A., Lewis S.E., Richards S., Ashburner M., Henderson S.N., Sutton G.G., Wortman J.R., Yandell M.D., Zhang Q., Chen L.X., Brandon R.C., Rogers Y.H., Blazej R.G., Champe M., Pfeiffer B.D., et al. 2000. The genome sequence of Drosophila melanogaster. Science 287: 2185 .

Allfrey V.G., Faulkner R., and Mirsky A.E. 1964. Acetylation and methylation of histones and their possible role in the regulation of RNA synthesis. Proc. Natl. Acad. Sci. 51: 786.

Bastow R., Mylne J.S., Lister C., Lippman Z., Martienssen R.A., and Dean C. 2004. Vernalization requires epigenetic silencing of FLC by histone methylation. Nature 427: 164.

Bird A. 2002. DNA methylation patterns and epigenetic memory. Genes Dev. 16: 6.

Brasher S.V., Smith B.O., Fogh R.H., Nietlispach D., Thiru A., Nielsen P.R., Broadhurst R.W., Ball L.J., Murzina N.V., and Laue E.D. 2000. The structure of mouse HP1 suggests a unique mode of single peptide recognition by the shadow chromo domain dimer. EMBO J. 19: 1587.

Briggs S.D., Bryk M., Strahl B.D., Cheung W.L., Davie J.K., Dent S.Y., Winston F., and Allis C.D. 2001. Histone H3 lysine 4 methylation is mediated by Set 1 and required for cell growth and rDNA silencing in Saccharomyces cerevisiae. Genes Dev. 15: 3286.

Cao R., Wang L., Wang H., Xia L., Erdjument-Bromage H., Tempst P., Jones R.S., and Zhang Y. 2002. Role of histone H3 lysine 27 methylation in Polycomb-group silencing. Science 298: 1039 .

Chadwick B.P. and Willard H.F. 2003. Chromatin of the Barr body: Histone and non-histone proteins associated with or excluded from the inactive X chromosome. Hum. Mol. Genet. 12: 2167 .

Czermin B., Melfi R., McCabe D., Seitz V., Imhof A., and Pirrotta V. 2002. Drosophila Enhancer of Zeste/ESC complexes have a histone H3 methyltransferase activity that marks chromosomal Polycomb sites. Cell 111: 185.

DeLange R.J. and Smith E.L. 1973. Histone 3. I. Isolation and sequences of the tryptic peptides from the maleylated calf thymus protein. J. Biol. Chem. 248: 3248.

Fang J., Feng Q., Ketel C.S., Wang H., Cao R., Xia L., Erdjument-Bromage H., Tempst P., Simon J.A., and Zhang Y. 2002. Purification and functional characterization of SET8, a nucleosomal histone H4-lysine 20-specific methyltransferase. Curr. Biol. 12: 1086.

Feng Q., Wang H., Ng H.H., Erdjument-Bromage H., Tempst P., Struhl K., and Zhang Y. 2002. Methylation of H3-lysine 79 is mediated by a new family of HMTases without a SET domain. Curr. Biol. 12: 1052.

Fischle W., Wang Y., and Allis C.D. 2003a. Histone and chromatin cross-talk. Curr. Opin. Cell Biol. 15: 172.

. 2003b. Binary switches and modification cassettes in histone biology and beyond. Nature 425: 475 .

Fischle W., Wang Y., Jacobs S.A., Kim Y., Allis C.D., and Khorasanizadeh S. 2003c. Molecular basis for the discrimination of repressive methyl-lysine marks in histone $\mathrm{H} 3$ by Polycomb and HP1 chromodomains. Genes Dev. 17: 1870.

Gasser S.M. and Cockell M.M. 2001. The molecular biology of the SIR proteins. Gene 279: 1.

Grunstein M. 1997. Molecular model for telomeric heterochromatin in yeast. Curr. Opin. Cell Biol. 9: 383.

Han J.S., Szak S.T., and Boeke J.D. 2004. Transcriptional disruption by the L1 retrotransposon and implications for mammalian transcriptomes. Nature 429: 268.

Jackson J.P., Johnson L., Jasencakova Z., Zhang X., PerezBurgos L., Singh P.B., Cheng X., Schubert I., Jenuwein T., and Jacobsen S.E. 2004. Dimethylation of histone H3 lysine 9 is a critical mark for DNA methylation and gene silencing in $\mathrm{Ara}$ bidopsis thaliana. Chromosoma 112: 308.

Jacobs S.A. and Khorasanizadeh S. 2002. Structure of HP1 chromodomain bound to a lysine 9-methylated histone $\mathrm{H} 3$ tail. Science 295: 2080.
Jaenisch R. and Bird A. 2003. Epigenetic regulation of gene expression: How the genome integrates intrinsic and environmental signals. Nat. Genet. 33: 245.

Jasencakova Z., Soppe W.J., Meister A., Gernand D., Turner B.M., and Schubert I. 2003. Histone modifications in Arabidopsis- high methylation of $\mathrm{H} 3$ lysine 9 is dispensable for constitutive heterochromatin. Plant J. 33: 471.

Jenuwein T. 2001. Re-SET-ting heterochromatin by histone methyltransferases. Trends Cell Biol. 11: 266.

Jenuwein T. and Allis C.D. 2001. Translating the histone code. Science 293: 1074.

Jones P.A. and Baylin S.B. 2002. The fundamental role of epigenetic events in cancer. Nat. Rev. Genet. 3: 415.

Kazazian H.H., Jr. 2004. Mobile elements: Drivers of genome evolution. Science 303: 1626.

Klochendler-Yeivin A., Muchardt C., and Yaniv M. 2002. SWI/SNF chromatin remodeling and cancer. Curr. Opin. Genet. Dev. 12: 73.

Kohlmaier A., Savarese F., Lachner M., Martens J.H., Jenuwein T., and Wutz A. 2004. A chromosomal memory triggered by Xist regulates histone methylation in $\mathrm{X}$ inactivation. PloS Biol. 2: 991.

Kouzarides T. 2002. Histone methylation in transcriptional control. Curr. Opin. Genet. Dev. 12: 198.

Kuzmichev A., Jenuwein T., Tempst P., and Reinberg D. 2004. Different EZH2-containing complexes target methylation of histone H1 or nucleosomal histone H3. Mol. Cell 14: 183.

Kuzmichev A., Nishioka K., Erdjument-Bromage H., Tempst P., and Reinberg D. 2002. Histone methyltransferase activity associated with a human multiprotein complex containing the Enhancer of Zeste protein. Genes Dev. 16: 2893.

Lachner M., O’Sullivan R.J., and Jenuwein T. 2003. An epigenetic road map for histone lysine methylation. J. Cell Sci. 116: 2117.

Luger K. 2003. Structure and dynamic behavior of nucleosomes. Curr. Opin. Genet. Dev. 13: 127.

Luger K., Mader A.W., Richmond R.K., Sargent D.F., and Richmond T.J. 1997. Crystal structure of the nucleosome core particle at 2.8 Å resolution. Nature 389: 251.

Maison C., Bailly D., Peters A.H., Quivy J.P., Roche D., Taddei A., Lachner M., Jenuwein T., and Almouzni G. 2002. Higherorder structure in pericentric heterochromatin involves a distinct pattern of histone modification and an RNA component. Nat. Genet. 30: 329.

Mateescu B., England P., Halgand F., Yaniv M., and Muchardt C. 2004. Tethering of HP1 proteins to chromatin is relieved by phosphoacetylation of histone H3. EMBO Rep. 5: 490.

Meehan R.R., Kao C.F., and Pennings S. 2003. HP1 binding to native chromatin in vitro is determined by the hinge region and not by the chromodomain. EMBO J. 22: 3164 .

Min J., Zhang Y., and Xu R.M. 2003. Structural basis for specific binding of Polycomb chromodomain to histone $\mathrm{H} 3$ methylated at Lys 27. Genes Dev. 17: 1823.

Muchardt C., Guilleme M., Seeler J.S., Trouche D., Dejean A., and Yaniv M. 2002. Coordinated methyl and RNA binding is required for heterochromatin localization of mammalian HP1alpha. EMBO Rep. 3: 975.

Müller J., Hart C.M., Francis N.J., Vargas M.L., Sengupta A., Wild B., Miller E.L., O'Connor M.B., Kingston R.E., and Simon J.A. 2002. Histone methyltransferase activity of a Drosophila Polycomb group repressor complex. Cell 111: 197.

Nakayama J., Rice J.C., Strahl B.D., Allis C.D., and Grewal S.I. 2001. Role of histone H3 lysine 9 methylation in epigenetic control of heterochromatin assembly. Science 292: 110.

Narlikar G.J., Fan H.Y., and Kingston R.E. 2002. Cooperation between complexes that regulate chromatin structure and transcription. Cell 108: 475.

Nielsen A.L., Oulad-Abdelghani M., Ortiz J.A., Remboutsika E., Chambon P., and Losson R. 2001. Heterochromatin formation in mammalian cells: Interaction between histones and HP1 proteins. Mol. Cell 7: 729 .

Nielsen P.R., Nietlispach D., Mott H.R., Callaghan J., Bannister A., Kouzarides T., Murzin A.G., Murzina N.V., and Laue E.D. 2002. Structure of the HP1 chromodomain bound to histone H3 methylated at lysine 9. Nature 416: 103. 
Nishioka K., Rice J.C., Sarma K., Erdjument-Bromage H., Werner J., Wang Y., Chuikov S., Valenzuela P., Tempst P., Steward R., Lis T.J., Allis C.D., and Reinberg D. 2002. PR-Set7 is a nucleosome-specific methyltransferase that modifies lysine 20 of histone $\mathrm{H} 4$ and is associated with silent chromatin. Mol. Cell 9: 1201.

Noma K. and Grewal S.I. 2002. Histone H3 lysine 4 methylation is mediated by Set 1 and promotes maintenance of active chromatin states in fission yeast. Proc. Natl. Acad. Sci. 99: 16438.

Noma K., Allis C.D., and Grewal S.I. 2001. Transitions in distinct histone $\mathrm{H} 3$ methylation patterns at the heterochromatin domain boundaries. Science 293: 1150.

O'Carroll D., Scherthan H., Peters A.H., Opravil S., Haynes A.R., Laible G., Rea S., Schmid M., Lebersorger A., Jerratsch M., Sattler L., Mattei M.G., Denny P., Brown S.D., Schweizer D., and Jenuwein T. 2000. Isolation and characterization of Suv39h2, a second histone $\mathrm{H} 3$ methyltransferase gene that displays testis-specific expression. Mol. Cell. Biol. 20: 9423.

Ogawa H., Ishiguro K., Gaubatz S., Livingston D.M., and Nakatani Y. 2002. A complex with chromatin modifiers that occupies E2F- and Myc-responsive genes in G0 cells. Science 296: 1132.

Okamoto I., Otte A.P., Allis C.D., Reinberg D., and Heard E. 2004. Epigenetic dynamics of imprinted X inactivation during early mouse development. Science 303: 644.

Paik W.K. and Kim S. 1971. Protein methylation. Science 174: 114.

Perez-Burgos L., Peters A.H., Opravil S., Kauer M., Mechtler K., and Jenuwein T. 2004. Generation and characterization of methyl-lysine histone antibodies. Methods Enzymol. 376: 234.

Peters A.H., Kubicek S., Mechtler K., O'Sullivan R.J., Derijck A.A., Perez-Burgos L., Kohlmaier A., Opravil S., Tachibana M., Shinkai Y., Martens J.H., and Jenuwein T. 2003. Partitioning and plasticity of repressive histone methylation states in mammalian chromatin. Mol. Cell 12: 1577.

Plath K., Fang J., Mlynarczyk-Evans S.K., Cao R., Worringer K.A., Wang H., de la Cruz C.C., Otte A.P., Panning B., and Zhang Y. 2003. Role of histone H3 lysine 27 methylation in $\mathrm{X}$ inactivation. Science 300: 131.

Rea S., Eisenhaber F., O'Carroll D., Strahl B.D., Sun Z.W., Schmid M., Opravil S., Mechtler K., Ponting C.P., Allis C.D., and Jenuwein T. 2000. Regulation of chromatin structure by site-specific histone H3 methyltransferases. Nature 406: 593.

Rice J.C., Nishioka K., Sarma K., Steward R., Reinberg D., and Allis C.D. 2002. Mitotic-specific methylation of histone H4 Lys 20 follows increased PR-Set7 expression and its localization to mitotic chromosomes. Genes Dev. 16: 2225.

Rice J.C., Briggs S.D., Ueberheide B., Barber C.M., Shabanowitz J., Hunt D.F., Shinkai Y., and Allis C.D. 2003. Histone methyltransferases direct different degrees of methylation to define distinct chromatin domains. Mol. Cell 12: 1591 .

Santos F., Zakhartchenko V., Stojkovic M., Peters A., Jenuwein T., Wolf E., Reik W., and Dean W. 2003. Epigenetic marking correlates with developmental potential in cloned bovine preimplantation embryos. Curr. Biol. 13: 1116.

Santos-Rosa H., Schneider R., Bannister A.J., Sherriff J., Bernstein B.E., Emre N.C., Schreiber S.L., Mellor J., and Kouzarides T. 2002. Active genes are tri-methylated at K4 of histone H3. Nature 419: 407.

Schneider R., Bannister A.J., and Kouzarides T. 2002. Unsafe SETs: Histone lysine methyltransferases and cancer. Trends Biochem. Sci. 27: 396.

Schotta G., Lachner M., Sarma K., Ebert A., Sengupta R., Reuter G., Reinberg D., and Jenuwein T. 2004. A silencing pathway to induce H3-K9 and H4-K20 trimethylation at constitutive heterochromatin. Genes Dev. 18: 1251.

Silva J., Mak W., Zvetkova I., Appanah R., Nesterova T.B., Webster Z., Peters A.H., Jenuwein T., Otte A.P., and Brockdorff N. 2003. Establishment of histone H3 methylation on the inactive $\mathrm{X}$ chromosome requires transient recruitment of Eed-Enx1 polycomb group complexes. Dev. Cell 4: 481.
Strahl B.D. and Allis C.D. 2000. The language of covalent histone modifications. Nature 403: 41.

Strahl B.D., Grant P.A., Briggs S.D., Sun Z.W., Bone J.R., Caldwell J.A., Mollah S., Cook R.G., Shabanowitz J., Hunt D.F., and Allis C.D. 2002. Set2 is a nucleosomal histone H3-specific methyltransferase that mediates transcriptional repression. Mol. Cell. Biol. 22: 1298.

Sung S. and Amasino R.M. 2004. Vernalization in Arabidopsis thaliana is mediated by the PHD finger protein VIN3. Nature 427: 159 .

Tachibana M., Sugimoto K., Nozaki M., Ueda J., Ohta T., Ohki M., Fukuda M., Takeda N., Niida H., Kato H., and Shinkai Y. 2002. G9a histone methyltransferase plays a dominant role in euchromatic histone $\mathrm{H} 3$ lysine 9 methylation and is essential for early embryogenesis. Genes Dev. 16: 1779.

Tamaru H., Zhang X., McMillen D., Singh P.B., Nakayama J., Grewal S.I., Allis C.D., Cheng X., and Selker E.U. 2003. Trimethylated lysine 9 of histone H3 is a mark for DNA methylation in Neurospora crassa. Nat. Genet. 34: 75.

Turner B.M. 2000. Histone acetylation and an epigenetic code. Bioessays 22: 836.

van Holde K.E. 1988. Chromatin. Springer Verlag, New York. van Leeuwen F., Gafken P.R., and Gottschling D.E. 2002. Dotlp modulates silencing in yeast by methylation of the nucleosome core. Cell 109: 745.

Volpe T., Schramke V., Hamilton G.L., White S.A., Teng G., Martienssen R.A., and Allshire R.C. 2003. RNA interference is required for normal centromere function in fission yeast. Chromosome Res. 11: 137

Waterston R.H., Lindblad-Toh K., Birney E., Rogers J., Abril J.F., Agarwal P., Agarwala R., Ainscough R., Alexandersson M., An P., Antonarakis S.E., Attwood J., Baertsch R., Bailey J., Barlow K., Beck S., Berry E., Birren B., Bloom T., Bork P., Botcherby M., Bray N., Brent M.R., Brown D.G., and Brown S.D., et al. (Mouse Genome Sequencing Consortium). 2002. Initial sequencing and comparative analysis of the mouse genome. Nature 420: 520.

Watson J.D. (Interview). 2003. Celebrating the genetic jubilee: A conversation with James D. Watson. Sci. Am. (April issue) 2003: 67.

Wood V., Gwilliam R., Rajandream M.A., Lyne M., Lyne R., Stewart A., Sgouros J., Peat N., Hayles J., Baker S., Basham D., Bowman S., Brooks K., Brown D., Brown S., Chillingworth T., Churcher C., Collins M., Connor R., Cronin A., Davis P., Feltwell T., Fraser A., Gentles S., and Goble A., et al. 2002. The genome sequence of Schizosaccharomyces pombe. Nature 415: 871 .

Xiao B., Jing C., Wilson J.R., Walker P.A., Vasisht N., Kelly G., Howell S., Taylor I.A., Blackburn G.M., and Gamblin S.J. 2003. Structure and catalytic mechanism of the human histone methyltransferase SET7/9. Nature 421: 652.

Xiao T., Hall H., Kizer K.O., Shibata Y., Hall M.C., Borchers C.H., and Strahl B.D. 2003. Phosphorylation of RNA polymerase II CTD regulates H3 methylation in yeast. Genes Dev. 17: 654 .

Yang L., Xia L., Wu D.Y., Wang H., Chansky H.A., Schubach W.H., Hickstein D.D., and Zhang Y. 2002. Molecular cloning of ESET, a novel histone H3-specific methyltransferase that interacts with ERG transcription factor. Oncogene 21: 148.

Yeast Genome Directory. 1997. The yeast genome directory. Nature (suppl. 6632) 387: 5.

Zhang L., Eugeni E.E., Parthun M.R., and Freitas M.A. 2003. Identification of novel histone post-translational modifications by peptide mass fingerprinting. Chromosoma 112: 77.

Zhang X., Yang Z., Khan S.I., Horton J.R., Tamaru H., Selker E.U., and Cheng X. 2003. Structural basis for the product specificity of histone lysine methyltransferases. Mol. Cell 12: 177.

Zhang Y. and Reinberg D. 2001. Transcription regulation by histone methylation: Interplay between different covalent modifications of the core histone tails. Genes Dev. 15: 2343. 


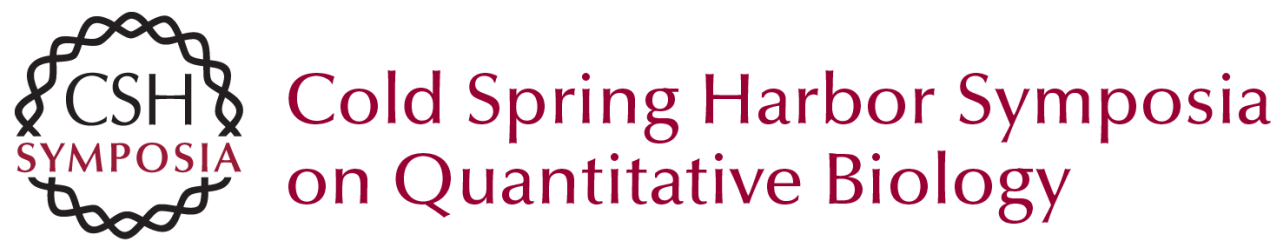

\section{Trilogies of Histone Lysine Methylation as Epigenetic Landmarks of the Eukaryotic Genome}

M. LACHNER, R. SENGUPTA, G. SCHOTTA, et al.

Cold Spring Harb Symp Quant Biol 2004 69: 209-218

Access the most recent version at doi:10.1101/sqb.2004.69.209

References This article cites 80 articles, 30 of which can be accessed free at: http://symposium.cshlp.org/content/69/209.full.html\#ref-list-1

\section{License}

Email Alerting

Receive free email alerts when new articles cite this article - sign up in Service the box at the top right corner of the article or click here. 